\title{
RELIABLE DETECTION OF SIGNAL TONES IN PRESENCE OF COLLISIONS*
}

\author{
$U D C((621.931: 004): 517.44)$
}

\begin{abstract}
Milica Jovanović, Igor Stojanović, Sandra Đošić, Goran Đorđević
University of Niš, Faculty of Electronic Engineering, Department of Electronic, Niš, Republic of Serbia
\end{abstract}

\begin{abstract}
A reliable and energy-efficient contention resolution mechanism (CRM) is a crucial component of MAC protocols for wireless sensor networks (WSNs). Among a number of CRM proposals, CRMs based on exchanging short signal tones between competing nodes have recently drawn attention due to their promise of collisionfreedom and energy-efficiency. However, the basic assumption of these protocols, i.e. the possibility to reliably detect the presencelabsence of a signal tone in case of simultaneous transmissions from multiple nodes, has not yet been confirmed. In this paper a technique for signal tone generation/detection is presented, which uses only standard features of off-the-shelf $R F$ transmitters. Furthermore, the results of an experimental validation carried out in a real testbed are presented which demonstrates a high accuracy of signal tone detection in the presence of multiple simultaneous transmissions.
\end{abstract}

Key words: wireless sensor networks, energy efficiency, intra-cluster communication, signal tones

\section{INTRODUCTION}

High energy efficiency and prolonged network lifetime in large-scale wireless sensor networks (WSNs) [3] can be achieved by grouping spatially close sensor nodes into disjoint subsets called clusters [1][2][3]. Each cluster consists of a dedicated sink node, called cluster head $(\mathrm{CH})$, and multiple cluster members $(\mathrm{CMs})$. $\mathrm{CH}$ collects and aggregates sensed data from CMs, via intra-cluster communication and then, it forwards the aggregated data to the base station, directly or in a multi-hop manner involving CHs of intermediate clusters. A number of MAC protocols are proposed for both intra-cluster

Received November, 23, 2018

Corresponding author: Milica D. Jovanović

Faculty of Electronic Engineering, Aleksandra Medvedeva 14, 18000 Niš, Republic of Serbia

E-mail: milica.jovanovic@elfak.ni.ac.rs

* Acknowledgement: This research was sponsored in part by the Serbian Ministry of Science and Technological Development, project no. TR-32009 and in part by project no. TR-33051 
communication (between $\mathrm{CH}$ and $\mathrm{CM}$ ) and inter-cluster communication (between $\mathrm{CHs}$ ) [4][5]. The energy efficiency of the CMs is mostly affected by an intra-cluster MAC protocol, which manages the CMs access to the shared medium and tries to ensure that no collisions occur [6].

Intra-cluster MAC protocols can be classified as TDMA-based and contention-based protocols. TDMA-based MAC protocols divide time into frames of fixed duration. Frames are divided into slots, and each CM is assigned one slot in the frame. TDMAbased protocols are characterized by low control overhead, but they are also inflexible in the sense that a CM cannot utilize slots of others, currently inactive, CMs to transfer a larger data packet to $\mathrm{CH}$ in the same frame. In contention-based protocols, sensor nodes coordinate their active/sleep periods such that all nodes are awake at the same time. During the active period, CMs first contend for medium access, by using a suitable contention resolution mechanism (CRM), and then, the winner of the contention transmits the data packet to $\mathrm{CH}$.

CRM based on the carrier sense multiple access (CSMA) protocol is often chosen due to its simplicity, low contention overhead and scalability [7]. With this scheme, the contention period is divided into a number of short contention slots. CM randomly selects a contention slot, senses the channel and withdraws if the channel is busy; otherwise it announces upcoming data transmission by transmitting a carrier signal until the end of the contention period. However, CSMA is susceptible to hidden terminal collisions, which cause high energy wastage due to the transmission and reception of collided data packets, as well as retransmission of lost data. The RTS/CTS handshake mechanism solves the hidden terminal problem and is widely used in general wireless networks [8]. With RTS/CTS, CM informs CH of its intent to transmit by sending a request-to-send (RTS) control packet. Then, it waits for clear-to-send (CTS) control packet from $\mathrm{CH}$ before starting the transmission of the data packet. Although the use of RTS/CTS mechanism avoids most of the collisions, it is particularly unsuitable for WSNs because of high overhead due to control packets exchange [9].

A special class of CRMs, based on the binary countdown protocol (BCD) [10], replaces RTS/CTS handshake with the exchange of RF tones (called signal tones) among neighbouring nodes to avoid collisions of data packets in WSN [11][12][12]. The signal tone is a short burst of RF energy transmitted on the channel frequency. By using a standard packet-oriented RF transceiver (e.g. CC1101 [14]), a signal tone is typically implemented as a short preamble-only packet. With respect to RTS/CTS control packets, the signal tones can carry binary information, only, but it is much shorter and thus consumes less energy. In the BCD protocol, contentions are resolved in several elimination rounds. At each round, a subgroup of nodes transmits a signal tone, while all the other nodes listen to the channel, and give up their attempt of gaining access to the channel if the channel is busy, otherwise, they proceed to the next round. In our earlier work, we proposed a BCD-like contention resolution algorithm with tone-based signalling in TDMA-based WSNs [15]. In [16] we proposed STAR-TONE MAC protocol, with bit map - binary countdown (BM-BCD) CRM, which modifies the baseline BCD scheme in a following way. Firstly, STAR-TONE involves CH actively into the contention resolution process, which eliminates the hidden terminal problem. Secondly, STAR-TONE introduces a novel group-splitting algorithm which allows extension of the contention period with additional rounds, which lowers the number of tone transmissions. The performance advantage of STAR-TONE protocol is particularly 
evident under bursty traffic load conditions, when the contention resolution mechanism has to deal with a large number of contending nodes, and when it significantly lowers the energy consumption while increases the throughput.

The crucial assumption with tone-based MAC protocols is that $\mathrm{CH}$ is able to reliably detect the presence of a signal tone even in case of multiple simultaneous tone transmissions. In order to prove this assumption, in this paper we develop the method for signal tone generation and detection, using only standard features of off-the-shelf RF transmitters. In addition, we report the results of experiments with real sensor nodes, which confirm the feasibility of tone-based CRM.

The rest of the paper is organized as follows. Section 2 gives the short overview of the previously proposed STAR-TONE protocol [16]. Section 3 proposes the method for generation and detection of the signal tones. Section 5 describes the experimental setup and the performance results obtained. Conclusions are drawn in Section 6.

\section{STAR-TONE PROTOCOL}

Before providing a detailed analysis of the method for generation and detection of signal tones, we will first give a brief overview of the STAR-TONE protocol [16]. STAR-TONE is a tone-based contention resolution protocol for clustered WSNs of star topology. The communication within the cluster is performed in a tightly synchronized manner with time divided into time-slots, and each slot accommodates one data packet transmission. The slots are not preassigned to individual CMs. Instead, potential senders (i.e. the CMs with data to send) must contend for medium access at the beginning of each slot and only the winner of the competition can send its data to $\mathrm{CH}$.

STAR-TONE is based on a multi-round elimination process, which divides the set of CMs recursively into two subgroups, eliminates one subgroup and continues the procedure until a subgroup is of size 1 . At the beginning of each contention round, the set of noneliminated CMs is split into two groups: the group of active contenders (AC), and the group of silent contenders (SC). If an AC group is not empty (i.e. it contains of at least one potential sender), the SC group is eliminated, and all potential senders in this group are instructed to withdraw from the competition. Otherwise, the algorithm eliminates the $\mathrm{AC}$ group and keeps the SC group. The algorithm finishes when the survived group of CMs contains one node only, which becomes the winner of the contention.

The contention resolution process of the STAR-TONE protocol is illustrated in Fig 1. In this example four of the seven CMs are potential senders (represented with black dots). In the first round (CR 0), the $\mathrm{AC}$ group consists of three $\mathrm{CMs}$, among which two are potential senders. Since there are potential senders in AC group, the SC group is eliminated, which means that all potential senders from this group withdrawn from the contention in this round. In the second round (CR 1), the AC group consists of only one CM, which is not a potential sender. Thus, the $\mathrm{AC}$ group is eliminated and all $\mathrm{CMs}$ in the $\mathrm{SC}$ group proceed to the next round. In the third round (CR 2), both groups consist of only one CM. Since CM from the AC group is a potential sender, the SC group is eliminated. As the AC group consists of only one $\mathrm{CM}$, and it is a potential sender, this $\mathrm{CM}$ is also the winner of the competition.

The two main components of STAR-TONE are: (a) the tone-based signalling mechanism, which is used to test the presence or absence of potential senders in the AC 
group and present this information to potential senders in the SC group; and (b) the group splitting algorithm, which partitions the group of non-eliminated CMs locally (at CMs) into $\mathrm{AC}$ and SC groups at the beginning of each contention round.
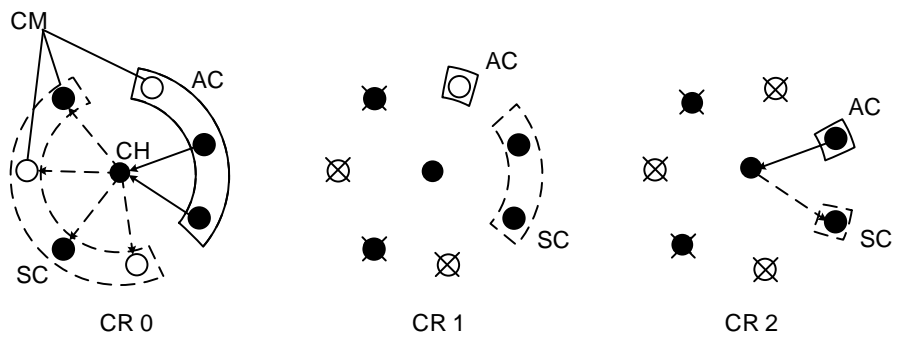

Fig. 1 Example of the STAR-TONE CRM in a cluster of $7 \mathrm{CMs}$

\subsection{Tone-based signalling mechanism}

The contention period of the STAR-TONE protocol consists of $M$ contention rounds, and each round is divided into two mini slots as shown in Fig 2. In this figure, shaded rectangles represent the tone transmissions while unshaded ones represent the tone detections. Each round has assigned an index $r$, starting from 0 . In the first mini-slot of a round, potential senders from the $\mathrm{AC}$ group announce their presence to $\mathrm{CH}$ by transmission of the transmitter-tone (T-tone). In the second mini-slot, $\mathrm{CH}$ notifies the potential senders from the SC group about the presence (or absence) of potential senders in the $\mathrm{AC}$ group, by transmitting (or not transmitting) the receiver-tone ( $R$-tone). If a silent potential sender receives an R-tone it will withdraw from the competition (because it knows that there is at least one potential sender in the AC group). Otherwise, it will proceed to the next round.

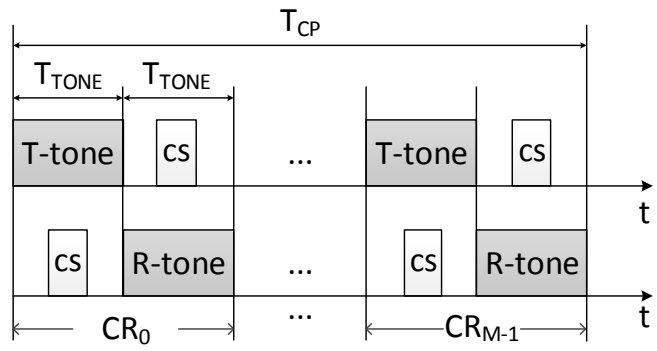

Fig. 2 Contention period

Receiving a signal tone means detecting the presence of an RF signal, so, in order to receive a signal tone, $\mathrm{CM}$ ( or $\mathrm{CH}$ ) should sample the channel at the middle of mini-slot. The basic assumption of a tone-based signalling mechanism is that the tone collision does not violate their functionality. If a T-tone transmission is interpreted as logical 1 , and the absence of T-tone transmission as logical 0 , than during the first mini-slot of a contention round $\mathrm{CH}$ should receive the 'logical OR' of the T-tone transmissions of all potential senders in the AC group. 


\section{SignAL TONES}

Signal tones are used in STAR-TONE as a means of signalling between transmitters and receivers during the contention resolution process. The signal tone is a short-lasting modulated or unmodulated RF signal transmitted on the channel frequency. Duration of the signal tone has to be short, in order to achieve time and energy efficiency, but long enough to enable reliable detection even in the presence of clock drift and radio interference.

Signal tone carries the binary information, i.e. the signal is present or absent. Therefore there is no need for RF tone demodulation at the receiver. The presence of the signal tone can be detected by measuring the strength of the receiving signal, i.e. by sampling the channel. However, in the case of simultaneous transmissions, as shown in Fig. 3, the superposition of $n$ overlapping signal tones creates a compound signal, whose strength depends on several factors, such as the relative strength and phase offset of individual signal tones. Therefore, the main question this paper seeks to resolve is whether the measurement of the signal strength is still a reliable method of signal tone detection in the presence of multiple simultaneous tone transmissions.

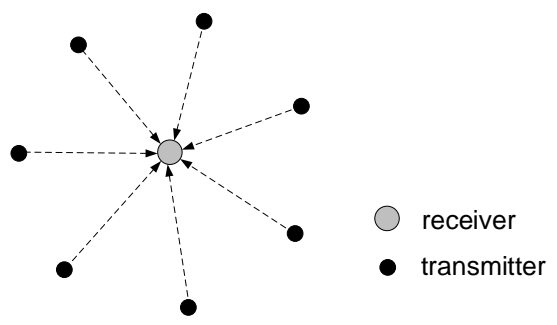

Fig. 3 Multiple signal tones reception

To this end, we will first describe a method for generation and detection of signal tones that uses standard features of off-the-shelf RF transmitters. Unfortunately, they do not provide a built-in support for generation and detection of signal tones. However, with most of them, signal tones can be generated/detected in an indirect way, by using available functions of the receiver. For our experimentations we use the transceiver CC1101 [14], and all the techniques for the generation and detection of signal tones are adapted to it. These techniques can easily be adapted to most of other RF transceivers for WSNs with minor modifications.

\subsection{Development platform}

For development and validation of proposed techniques we use the wireless sensor node platform based on the Texas Instrument's RF System-on-chip, CC430, which incorporates MPS430 CPU and CC1101 RF transceiver, together with the set of standard CPU peripherals [17].

Mixed-signal CPUs from the MSP430 family are based on CPU 16-bit RISC architecture and optimized for low cost and low power consumption. The current in the idle mode is lower than $1 \mu \mathrm{A}$. The highest CPU speed is $25 \mathrm{MHz}$. The wake-up latency is below $1 \mu \mathrm{s}$, which allows CPU to stay in sleep mode longer, and minimizes the 
consumption. Various peripherals can work autonomously due to low level interrupts, which also minimizes the time that CPU spends in active mode.

CC1101 is a low-range sub-gigahertz RF transceiver designed for very low-power wireless applications. It can be configured for operation at different frequencies in the 300-348 MHz, 387-464 MHz and 779-928 MHz bands. The RF transceiver is integrated with a highly configurable baseband modem. The modem supports various modulation formats and has a configurable data rate up to $600 \mathrm{kbps}$. Operating modes of transceiver are IDLE, SLEEP, TRANSMIT and RECEIVE. IDLE is the initial state of the transceiver, with typical current consumption of $1.7 \mathrm{~mA}$. SLEEP is the lowest consumption mode, typically $200 n A$, with the transceiver turned off and the current state of the most of the registers conserved. In the TRANSMIT state either the packet from the transmit buffer or the continuous preamble is transmitted, with a typical consumption of $16.8 m A-34.2 m A$, depending on the output power, or the continuous preamble is transmitted, if the transmit buffer is empty. During the RECEIVE state the reception of the pocket is performed, with typical consumption of $14.7 \mathrm{~mA}$.

C1101 provides the extensive hardware support for packet handling, data buffering, burst transmissions, clear channel assessment, link quality indication, and wake-on-radio. The built-in support for packet-oriented protocols simplifies the transceiver-CPU interaction, and frees up CPU from low-level activities. The task of CPU is to prepare the packet in its local memory, copy the content of the packet to the transmit buffer of the transmitter, and issue the transmit command, as shown in Fig. 4. In the RECEIVE state, the transceiver demodulates the received RF signal and puts received data bits in the receive buffer. After the whole packet is received, the transceiver informs CPU via interrupt, which then copies the packet in its local memory.

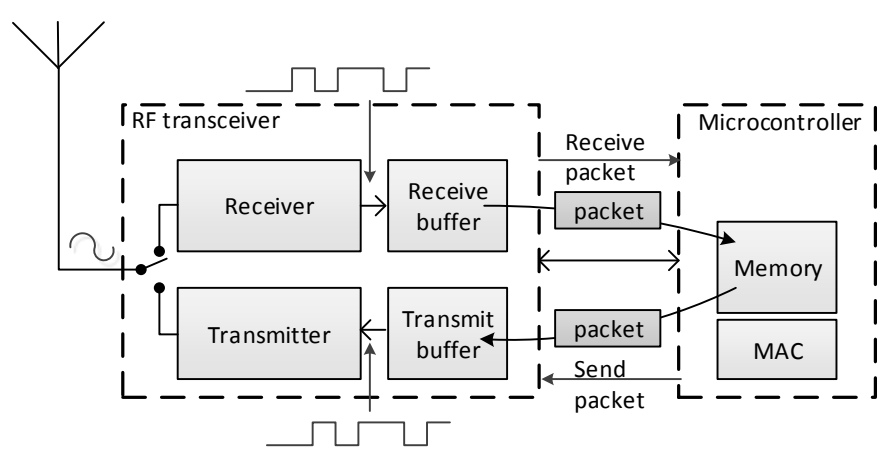

Fig. 4 Packet exchange between CPU and RF transceiver.

The packet format for the CC1101 transceiver is shown in Fig. 5. Some parts of this packet can be configured. The packet consists of:

- Preamble: series of alternating zeroes and ones. The preamble length, given in number of bytes, can be configured, and its minimum value is 1 byte.

- Synchronization word: indicates the end of preamble and beginning of the payload data. Its default length is 2 bytes, and can be extended to 4 bytes.

- Length: information of the length of the data field. It is used only in the mode with the variable data length. 
- Address: address of the receiver node.

- Data: contains user data. The minimum length of this field is 1 byte.

- CRC-16: control sum calculated from fields Length, Address and Data; this field is 2 bytes.

\begin{tabular}{|c|c|c|c|c|c|}
\hline $\begin{array}{c}\text { Preamble } \\
(1010 . .1010)\end{array}$ & $\begin{array}{c}\text { Synchronization } \\
\text { word }\end{array}$ & Length & Address & Data & CRC-16 \\
\hline $\begin{array}{c}\mathrm{N}_{\text {pre }} \\
(8 \times \mathrm{x} \text { bitov })\end{array}$ & $\begin{array}{c}\mathrm{N}_{\text {synch }} \\
(16 / 32 \mathrm{bit})\end{array}$ & $\begin{array}{c}\mathrm{N}_{\text {length }} \\
(8 \mathrm{bit})\end{array}$ & $\begin{array}{c}\mathrm{N}_{\text {addr }} \\
(8 \mathrm{bit})\end{array}$ & $\begin{array}{c}\mathrm{N}_{\text {data }} \\
(8 \mathrm{x} \mathrm{n} \text { bit })\end{array}$ & $\begin{array}{c}\mathrm{N}_{\text {crc }} \\
(16 \mathrm{bit})\end{array}$ \\
\hline
\end{tabular}

Fig. 5 Pocket format for $\mathrm{CC} 1101$ transceiver

Preamble, synchronization word and CRC-16 are automatically added by the transmitter and removed by the receiver.

\subsection{Signal tone generation}

The basic requirement for signal tone generation is the possibility for accurate regulation of tone duration. However, since the CC1101 only operates in packet mode, it is impossible to generate a signal tone by directly controlling the transmitter circuitry, so the indirect way of signal tone duration regulation is needed. There are two approaches on how to generate single tones with CC1101.

Packet-tone. In this approach an RF tone can be generated in the form of a regular packet of pre-specified length and arbitrary content. For a packet of total length of $N_{p k t}$, the tone duration is:

$$
T_{\text {TONE }}=\frac{N_{p k t}}{\text { bit_rate }}
$$

Assuming that preamble, the synchronization word and CRC-16 fields are all configured with the minimum length, the length of packet can be calculated as $N_{p k t}=$ $(5+N)$ bytes, where $N \geq 1$ is the length of the data field, in bytes. Since the minimum length of this field is $1 \mathrm{~B}$, the minimum tone duration is $T_{T O N E}=2.5 \mathrm{~ms}$ assuming bit rate of $19.2 \mathrm{~kb} / \mathrm{s}$. Since the length of data field is an integer value, the signal tone duration must also be given in discreet units with the quantization step of $0.42 \mathrm{~ms}$. The advantage of this method is the possibility of precise tone duration regulation, and the shortcoming is a relatively high CPU engagement, which has to load the transmit buffer with the packet content for each signal tone, as well as the relatively high minimum duration of the signal tone and coarse-grained quantization step.

Preamble-tone. The alternative way to generate a signal tone is to use the feature of CC1101 to transmit an uninterrupted preamble as long as the transmit buffer is empty. The signal tone is generated in the following manner: CPU enables transmission (which forces $\mathrm{CC} 1101$ to start preamble transmission) and starts the timer with timeout adjusted to the signal tone duration. After the timer timeouts, CPU disables transmission, which stops the preamble transmission. At all times the transmit buffer remains empty. This method is convenient because it allows both transmission of short signal tones and small quantization step with minimum CPU engagement. The shortcoming is the uncertainty in 
signal tone duration, due to the latency of the CPU interrupts. However, the advantages are higher than the shortcomings, so we chose this method for signal tone generation.

\subsection{Signal tone detection}

A crucial issue with signal tone based MAC protocols is to provide a reliable detection of signal tones. The detection method should be fast (in order to allow detection of short-lasting signal tones), energy-efficient and interference-free (since the interference is common in WSNs), while providing a low probability of false detections. Since the signal tone carries only binary information, the natural way for signal tone detection is to measure the strength of the received signal at the expected moment of signal tone transmission, and then compare the measured value with a threshold level. With this threshold-based signal detection method, the signal tone is detected (i.e. present) if the measured value is above the threshold level. Otherwise, the signal tone is considered to be absent. Most of the commercially available WSN transceivers have the support for measuring the strength of the received signal, usually in a form of the electronic circuitry commonly known as RSSI (Received Strength Signal Indicator). In CC1101, the RSS value is available in 8-bit RSSI register. The measured values for the RSS can be in range $[-138,-10.5] \mathrm{dBm}$ with the resolution of $0.5 \mathrm{dBm}$.

The procedure for signal tone detection is as follows:

1) CPU enables reception by switching transceiver's state from SLEEP to RECEIVE)

2) CPU reads RSSI after $2.4 \mathrm{~ms}$

3) CPU disables the reception by switching transceiver back to the SLEEP state.

Fig. 6 shows the current consumption of CC1101 during the channel sampling [17]. After CPU initiates reception, a time of $2.4 \mathrm{~ms}$ is required for $\mathrm{CC} 1101$ to transit from the SLEEP to the full RECEIVE state. Area marked as 1 in the Fig. 6 corresponds to the time needed for transition from the SLEEP to IDLE state. During the time period marked as 2, CC1101 calibrates internal circuitry, and then $2.4 \mathrm{~ms}$ after the reception is enabled, it enters the RECEIVE state. During the time period marked as 3, the CC1101 receiver is in an operating state. In this state, PLL is adjusted to the carrier frequency before the first RSS measurement is initiated. The first valid RSS measurement data is available in RSSI register at $t=3.3 \mathrm{~ms}$. After reading the RSSI register, CPU returns the transceiver to the SLEEP state (period 4). The whole channel sampling procedure lasts at minimum $3.7 \mathrm{~ms}$.

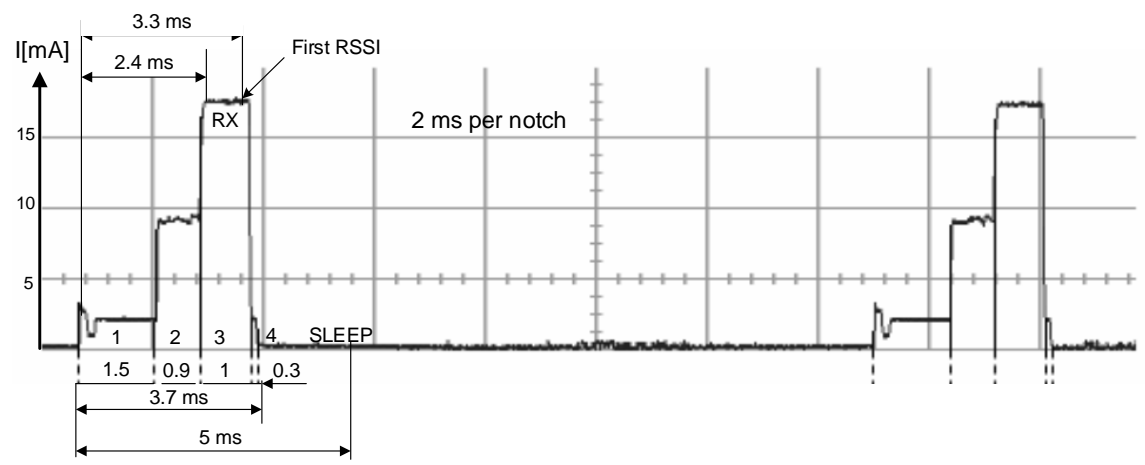

Fig. 6 CC1101 current consumption during the channel sampling. 
After the reception is disabled, $\mathrm{CC} 1101$ has to spend some time in the SLEEP state before the next reception is enabled. Thus, the minimum period for a single RSSI reading, including returning to the SLEEP state, is approximately $5 \mathrm{~ms}$.

\subsection{Signal tone duration}

A detailed analysis of the timing parameters from Fig. 6, allows us to estimate the basic signal tones time constraints. The duration of the channel sampling procedure ( $5 \mathrm{~ms}$ ) determines the minimum duration of the mini-slot (Fig. 2). The signal tone, which is generated by the transmitting node during the mini-slot should overlap with the time interval which starts at the moment when the receiver node enters the RECEIVE state $(t=2.4 \mathrm{~ms})$, and ends at the moment when it completes the RSS measurement $(3.3 \mathrm{~ms})$. Therefore, the minimum duration of a signal tone is, approximately, $\tau_{\min }=1 \mathrm{~ms}$.

Because in reality there is always some mismatch between the frequencies of the clocks in the transmitter and receiver nodes, the use of signal tone signalization requires time synchronization to adjust local clocks such that they match within prescribed limits. The methods for time synchronization in WSNs are out of the scope of this paper, and we only assume that a suitable time synchronization protocol is run periodically with a period of $T_{\text {sync }}$. If the clock drift rate of the nodes is $\delta p p m$ (parts per million), than the maximum clock drift between any two nodes in the network is $\theta=\delta T_{\text {sync }}$.

The signal tone duration $T_{T O N E}$ has to be long enough to provide the overlapping of the tone transmission with the channel sampling even in the case of maximum clock drift. The worst case scenario is created when the clock of one node delays for $\theta$ and of the other node precedes for $\theta$. Therefore, in order to guarantee the overlapping between the signal tone transmission and channel sampling, the signal tone transmission must start $2 \theta$ before and end $2 \theta$ after the channel sampling interval, as shown in Fig. 7. Thus, the signal tone duration should be (1):

$$
T_{\text {TONE }}=4 \theta+\tau_{\text {min }}=4 \delta T_{\text {sync }}+\tau_{\text {min }}
$$

where the $\tau_{\min }$ is the time needed for one channel sampling.

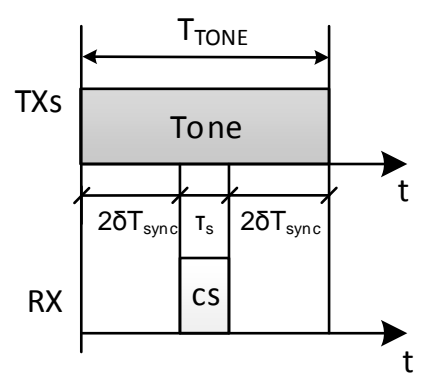

Fig. 7 Duration of the signal tone $\mathrm{T}_{\mathrm{TONE}}$ 


\section{EXPERIMENTAL RESULTS}

The basic assumption with tone-based MAC protocols for WSNs is the reliable detection of signal tones in the presence of collisions (i.e. multiple simultaneous tone transmissions). Usually, the collisions cause the corruption of the signals, which leads to the information loss. In contrast to this, the signal tone detection in tone-based MAC protocols is only concerned with the presence or absence of the signal, which is sensed by measuring RSS. Also, for proper operation of a tone-based MAC protocol, a receiving node should be able to detect the presence of the signal even if two or more nodes transmit their signal tones at the same time, i.e. the collision of signal tones should be non-destructive. Hence, the main concern with the signal tone detection mechanism is how the signal tone collisions affect the measured RSS value.

In order to confirm the assumption of the non-destructibility of the tone collisions, the following experiment has been conducted (Fig. 8). In this experiment we have used the development platform CC430, described in Section 3. The experimental setup consists of a central node, connected to the host computer, and five peripheral nodes, deployed in the circle of 15 meters in diameter. The central node periodically transmits broadcast messages, which are received by all peripheral nodes. With each message, the central node polls a subset of $k \in\{1, \ldots, 5\}$ peripheral nodes. After the reception of the broadcast message, the polled nodes simultaneously transmit the signal tone. The central node measures RSS and forwards this value to the host, which stores the RSS readings for later processing. This procedure has been repeated 1500 times for each subset of nodes.

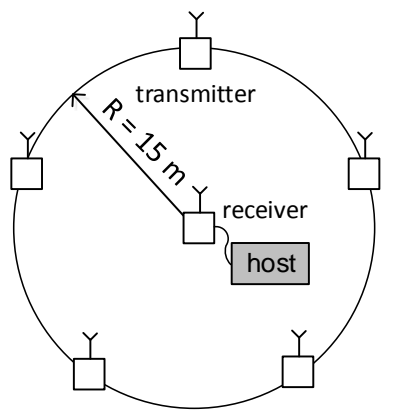

Fig. 8 Experimental setup: receiver and transmitter deployment

Figure 9 shows the RSSI data collected using the described procedure. Each graph in this figure shows the RSSI variations over time for $k=1,2,3,4,5$ active signal tone transmitters. In case of one active transmitter (Fig. 9(a)), the successive RSSI readings are relatively uniform. The RSSI value varies around $-71 \mathrm{dBm}$, with standard deviation of only $\pm 0.68 \mathrm{dBm}$. The RSSI variation is a consequence of ambient noise. In the case of two active transmitters Fig. 9(b)) the mean value varies around $-65 \mathrm{dBm}$, with standard deviation of $\pm 2 \mathrm{dBm}$. With the further increase of the number of simultaneously active transmitters (Fig. 9(c), Fig. 9(d) and Fig. 9(e)) there are two tendencies. Firstly, the mean value of RSSI increases, due to the higher total transmission energy. Secondly, the deviation of the measured values is higher, due to the interference of multiple RF signals at the receiver. These figures show that the measured RSS is always over $-72 \mathrm{dBm}$ 
(horizontal line in the pictures), regardless of the number of active transmitters. Therefore, we can conclude that it is safe to adopt the value of $-72 \mathrm{dBm}$ as the RSS threshold to differentiate between signal tone presence and absence.

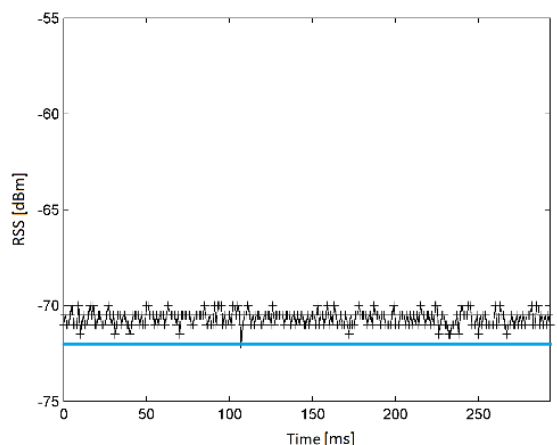

(a)

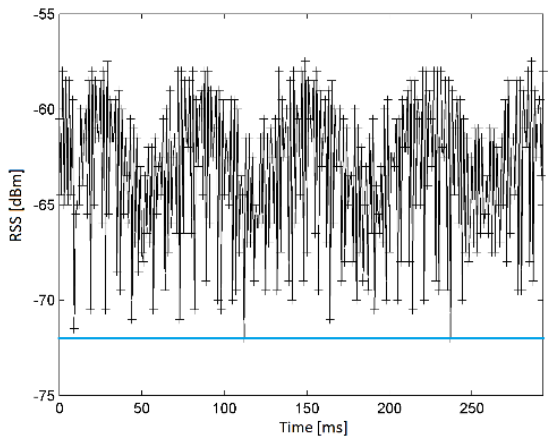

(c)

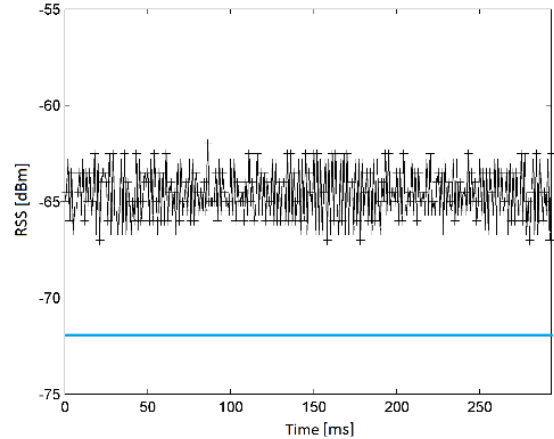

(b)

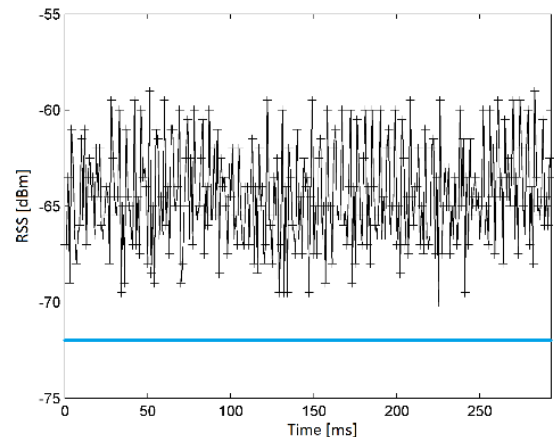

(d)

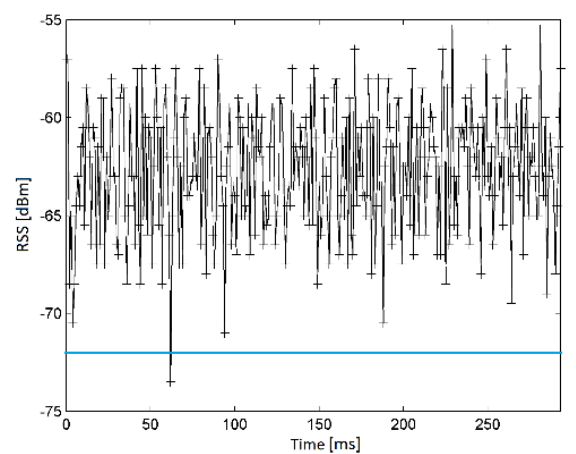

(e)

Fig. 9 Measured RSS values for different number of simultaneously active transmitters: (a) 1, (b) 2, (c) 3, (d) 4 and (e) 5 simultaneously active transmitters 
Table 1 shows the mean values $(\mu)$ and standard deviations $(\sigma)$ of RSSI for different number of simultaneously active transmitters. This table also shows the increase of the mean value with the increase of the number of simultaneously active transmitters, which compensates the increase of the standard deviation.

Table 1 Mean values and standard deviations of measured RSS

\begin{tabular}{ccc}
\hline $\begin{array}{c}\text { No. of the simultaneously } \\
\text { active transmitters }\end{array}$ & $\begin{array}{c}\text { Mean value } \\
{[\mathrm{dBm}]}\end{array}$ & $\begin{array}{c}\text { Standard deviation } \\
{[\mathrm{dBm}]}\end{array}$ \\
\hline 1 & $\mu=-71$ & $\sigma=0.68$ \\
2 & $\mu=-64.84$ & $\sigma=2.12$ \\
3 & $\mu=-64.02$ & $\sigma=3.47$ \\
4 & $\mu=-63.77$ & $\sigma=3.61$ \\
5 & $\mu=-63.23$ & $\sigma=3.34$ \\
\hline
\end{tabular}

The probability density functions of RSSI values for different number of active transmitters, and the corresponding cumulative distribution functions are shown in Fig. 10 and Fig. 11, respectively. Looking at the ranges of RSSI values in Fig. 10, we see that the distance between the minimum and maximum measured RSS values increases with the increase of the number of active transmitters. However, it should be noticed that the minimum values of these ranges are approximately the same, regardless of the number of active transmitters, with very few RSSI reading lower than $-75 \mathrm{dBm}$. These results indicate that the same threshold value as in the case of single transmitter can be used for detecting the presence of signal tone in case of multiple tone transmitters. The Fig. 11 also confirms the initial assumption that the RSS measurement provides reliable signal tone detection in presence of collisions, which justify the use of signal tones as a means for internode signalling during contention resolution phase of tone-based MAC protocols.

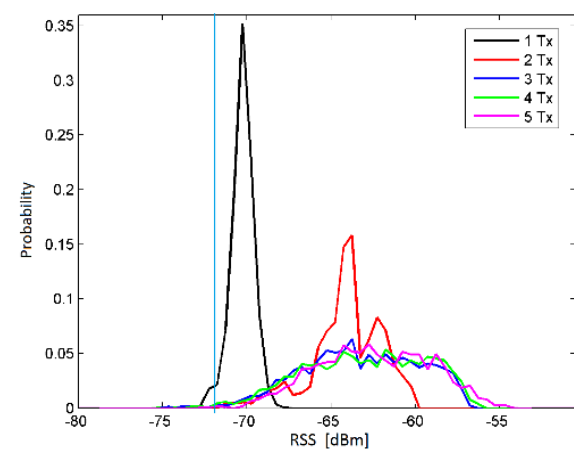

Fig. 10 RSSI values distribution

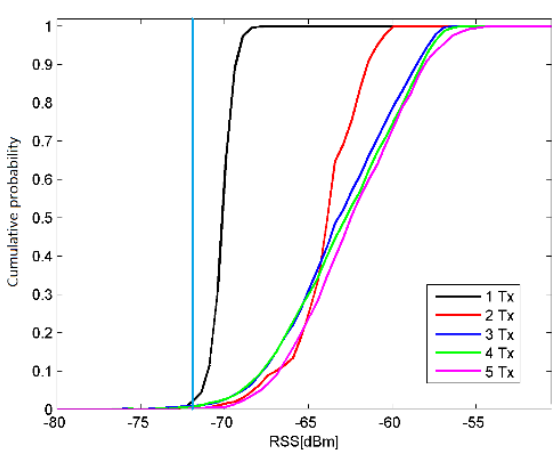

Fig. 11 Cumulative distribution function of RSSI values 


\section{CONCLUSION}

In this paper, we have investigated the feasibility of tone-based MAC protocols, in which the contentions are resolved by exchanging short RF signal tones between competing nodes. In particular, we have studied the STAR-TONE protocol, which is optimized for cluster networks, and provides a collision free communication between multiple peripheral nodes and singe cluster head. The contention resolution in the STARTONE protocol is divided into several two-phase elimination rounds, where all noneliminated peripheral nodes transmit their signal tones in the first phase, and the cluster head responds with a signal tone in the second phase. The crucial assumption with this protocol is that the cluster head is able to reliably detect the presence of a signal tone even in the case of multiple simultaneous tone transmissions. In order to test this assumption we have first proposed a method for signal tone generation and detection. In this method, the value of receiver signal strength indicator (RSSI) is used to distinguish between the signal tone presence/absence. Than we have conducted series of experiments to assess the performance of the detection method in the presence of multiple simultaneous tone transmissions. Experimental results confirm that the collision of signal tones does not affect the reliability of their detection. Our main observation is that the measured RSS value always stays above a threshold value, regardless of the number of active transmitters. In particular, because the increase of the number of active transmitters increases both the mean value and deviation of RSS values, the minimum measured RSS value stays practically the same as in the case of a single tone transmitter.

\section{REFERENCES}

[1] F.Akyildiz, Y. Sankarasubramaniam, W.Su, E.Cayirci, "Wireless sensor networks. A survey", Computer Networks, vol. 38, no. 3, pp. 393-422, 2002, doi:10.1109/WAINA.2009.192.

[2] G.Anastasi, M.Conti, M. Di Francesco, A. Passarella, "Energy Conservation in Wireless Sensor Networks: a Survey", Ad Hoc Networks, vol. 7, no. 3, pp. 537-568, 2009, doi: 10.1016/j.adhoc.2008.06.003

[3] Anadbabu, Siddaraju, R. Guru, "Energy Efficiency Mechanism in Wireless Sensor Networks: A Survey", International Journal of Computer Applications, vol. 139, no. 14, pp.27-33, 2016, doi: 10.5120/ijca2016908954.

[4] B. Mamalis, D. Gavalis, et al. "Clustering in Wireless Sensor Networks" in Y. Zhang, L. T. Yang, J. Chen (Ed.): 'RFID and Sensor Networks: Architectures, Protocols, Security, and Integrations', Boca Raton, CRC Press, 2010, pp. 323-353

[5] B. Priya, S.Solai Manohar, "Lifetime Enhancement of Cluster Based Wireless Sensor Network through Energy Efficient MAC Protocol", Circuits and Systems, vol. 7, no. 9, pp. 2296-2308, 2016, doi:10.4236/cs.2016.79200.

[6] V. Salmani, , H. C. Pai, "Intra-cluster Contention Resolution in Wireless Sensor Networks" in Multiple Access Communications, pp.170-181,vol. 7642 of Lecture Notes in Computer Science, Springer Berlin Heidelberg, 2012, doi: 10.1007/978-3-642-34976-8_18

[7] I. Demirkol, C. Ersoy, F. Alagoz, "MAC protocols for wireless sensor networks: A survey", IEEE Communications Magazine, vol. 44, no. 4, pp. 115-121, 2006, doi:10.1109/MCOM.2006.1632658.

[8] M. Sefuba, T. Walingo, F. Takawira, "Energy Efficient Medium Access Control Protocol for Clustered Wireless Sensor Networks with Adaptive Cross-Layer Scheduling”, Sensors (Basel), vol. 15, no. 9, pp. 24026-24053, 2015, doi: 10.3390/s150924026.

[9] A.Woo, D. E. Culler, "A transmission control scheme for media access in sensor networks", Proc. The 7 th annual international conference on mobile computing and networking (MOBICOM'07), July 2001, pp. 221-235, doi: 10.1145/381677.381699

[10] A.Tanenbaum, Computer Networks. Prentice Hall, NY, USA, 2003.

[11] D. Christmann, R. Gotzhein, S. Siegmund, F. Wirth, "Realization of Try-Once-Discard in Wireless Multihop Networks", IEEE transactions on Industrial Informatics, vol. 10, no. 1, pp. 17-26, 2014, doi: 10.1109/TII.2013.2281511. 
[12] N. Pereira, B. Andersson, E. Tovar, A. Rowe, "Static-priority scheduling over wireless networks with multiple broadcast domains", Proc. The 28th IEEE International Real-Time Systems Symposium (RTSS'07), Washington, DC, USA, 2007, pp. 447-458, doi: 10.1109/RTSS.2007.45.

[13] M. Jovanovic, I. Stojanovic, S. Đosić, G. Djordjevic, „Contention Resolution using Signal Tones for Wireless Sensor Networks", LII International Scientific Conference of Information, Communication and Energy Systems and Technologies, ICEST 2017,pp. 25-28, Nis, Serbia, June 2017, ISBN 978-86-6125$031-6$

[14] "CC1101 Low-Power Sub-1 GHz RF Transceiver", available at http://www.ti.com

[15] M. D. Jovanovic, G. Lj. Djordjevic, "Contention Resolution Mechanism for Receiver-Driven TDMABased Wireless Sensor Networks", Facta Universitatis, Series: Automatic Control and Robotics, vol. 12, no. 1, pp. 53-73. 2013.

[16] Milica D. Jovanovic, Igor Z. Stojanovic, Sandra M. Djosic, Goran Lj. Djordjevic, „Intra-Cluster Tone-Based Contention Resolution Mechanism for Wireless Sensor Networks", Computers and Electrical Engineering, Elsevier, Vol. 56, pp. 485-497, November 2016, DOI: 10.1016/j.compeleceng.2016.10.008, ISSN: 0045-7906, http://www.sciencedirect.com/science/article/pii/S0045790616305250

[17] „MSP430 ${ }^{\mathrm{TM}}$ SoC With RF Core”, available at http://www.ti.com

[18] „Application Note AN047“, available at http://www.ti.com 